\title{
ENCUADRE DESDE LA MIRADA DEL PSICOANÁLISIS RELACIONAL
}

Stella María Barrios Blanc Licenciada en Psicología, UDELAR Miembro Habilitante de AUDEPP Correo electrónico: barriosstella@gmail.com ORCID: 0000-0002-3201-7494 


\section{Resumen}

Desde la perspectiva del psicoanálisis relacional este trabajo aborda el encuadre y las distintas formas de estar con el paciente, tratando de indagar la peculiaridad que cada una de ellas conlleva. ¿Es posible sumergirse en todos los sentidos de lo que está pasando aquí, ahora, en mí, en el otro y entre los dos, en una interacción analítica a distancia?

El psicoanálisis relacional con sus ampliaciones conceptuales y sus referencias teóricas ofrece nuevas luces para observar estos fenómenos. El año 2020 inaugura algo distinto. La pandemia puso en crisis el encuadre clásico psicoanalítico. La propagación del covid-19, con sus medidas de confinamiento y sanitarias, hizo que se armaran distintos encuadres, que son distintos modos de encuentro con los pacientes. Comenzamos a utilizar distintas plataformas online para sostener y sostenernos frente a la irrupción de lo nuevo e inesperado que proviene del exterior y que es una amenaza para el encuentro presencial.

Aparece en nuestro trabajo la psicoterapia virtual en lugar de la presencial. Surgen interrogantes acerca de la forma en que estos dispositivos pueden facilitar o entorpecer el proceso psicoanalítico a la luz de las metapsicologías que nos acompañan.

Palabras clave: psicoanálisis relacional, encuadre, psicoanálisis online, neurociencia.

\section{The frame from a relational psychoanalytic point of view}

\section{Abstract}

From the perspective of relational psychoanalysis, I will approach the frame and the different ways of meeting the patient, trying to investigate the peculiarities that each of them holds. Is it possible to immerse oneself in every way into what is happening here, now and with me, with the other and between the two of us, in an analytical interaction at a distance?

Relational psychoanalysis and its conceptual extensions and theoretical references offers new insights to observe these phenomena.

In 2020 something different is revealed. The pandemic challenged the classical psychoanalytic frame. The spread of Covid-19 with its confinement and sanitary measures led to the creation of different frames, which are different ways of meeting the patients. We began to use different online platforms to support, both patients and ourselves, in the face of the irruption of something new and unexpected that comes from outside and that is a threat to face-to-face meetings.

Virtual psychotherapy replaces face-to-face psychotherapy. Questions arise as to how these arrangements can promote or hinder the psychoanalytic process considering the metapsychologies that accompany us.

Keywords: relational psychoanalysis, frame, online psychoanalysis, neuroscience. 


\section{PSICOANÁLISIS RELACIONAL: \\ UNA NUEVA MANERA DE PENSAR LO HUMANO}

El modelo relacional se fue gestando con los aportes de los cambios y avances sociales, culturales y científicos, que fueron haciendo rever los postulados clásicos del psicoanálisis, legados por su fundador. Manteniendo el espíritu investigador, no conformista, ha dado origen a nuevas maneras de pensar lo humano. El enfoque relacional en psicoanálisis

se enmarca en el intento de superar la dualidad clásica sujeto-objeto (interior-exterior), así como la descripción del comportamiento como una secuencia mecánica de acción y reacción, o agente y paciente. Desde la filosofía estas ideas son consonantes con el constructivismo, el perspectivismo (Nietzsche, Ortega), y el existencialismo en cuanto niega la preminencia de la esencia frente a la existencia. (Rodríguez Sutil, 2007, p. 9)

Con ese espíritu surge el psicoanálisis relacional, denominación propuesta por Stephen A. Mitchell (1946-2000), quien ha sido uno de los principales renovadores de la teoría psicoanalítica contemporánea y uno de los precursores de este giro relacional en psicoanálisis (Mitchell, 2015). El psicoanálisis relacional engloba varias líneas del pensamiento postclásico: los relacionalistas, los intersubjetivistas, los interpersonalistas (los teóricos de Boston Stern, Karlen Lyon-Ruth) y la teoría del apego de Bowlby (1988), que prioriza la necesidad básica de amor y exploración en el niño. Ellos comparten una premisa: se oponen a la metapsicología pulsional de Freud y enfatizan el papel de las relaciones en la constitución 
de la subjetividad. Lo intrapsíquico se entiende como producto de las relaciones y no como resultante de fuerzas endógenas. El ser humano nace en un ámbito relacional. Necesitamos de un otro para desarrollarnos, patologizarnos y curarnos. El otro es imprescindible.

El psicoanálisis relacional reformula el campo analítico, su proceso, su método y su teoría, revisada a la luz del estudio de lo intersubjetivo, los procesos vinculares y las investigaciones en neurociencias. Toma en cuenta el fenómeno de la neuroplasticidad, considerando el desarrollo de nuestro cerebro a lo largo de la vida y la posibilidad de incorporar nuevas actitudes de acuerdo con las nuevas experiencias (Ansermet y Magistretti, 2006). Al decir de Nemirovsky (2020),

pensar un sujeto abierto a su historia, a su presente político social y a su futuro, viviendo en esa paradoja planteada por esa ecuación repetición versus novedad y enfrentándonos con lo diverso, con lo que no es igual. Se abandona la idea central de la repetición, para dar lugar a lo nuevo, a lo que se edita, lo que aparece con el terapeuta, y desde allí se genera el cambio.

Conviven en este enfoque una amplia gama de conceptos de diversas escuelas psicoanalíticas que enfatizan aspectos como el self, el objeto o el espacio interpersonal entre el sí mismo y el otro. Concuerdan en el abandono del énfasis en la pulsión como elemento básico de la vida mental.

Se pasa de un modelo arqueológico y reconstructivo a una construcción activa de una narrativa de la vida del paciente. Paciente y analista son creadores de patrones (Aron, 2013). El psicoanálisis relacional considera que todos los fenómenos del proceso analítico se desarrollan en un campo cocreado entre paciente y terapeuta.

Los teóricos relacionales tienen en común tanto un interés en lo intrapsíquico como en lo interpersonal, y observan lo intrapsíquico como 
constituido por la internalización de la experiencia interpersonal, que a su vez está mediada por las limitaciones impuestas por las delimitaciones biológicas y los patrones (Aron, 2013). Asimismo, consideran que desempeñan roles importantes e interactivos en la vida humana: la realidad y la fantasía, lo interpersonal y lo intrapsíquico. Reconocen el valor de lo que trae el sujeto a la interacción: temperamento, eventos corporales, capacidad para responder fisiológicamente, patrones de regulación diferentes, sensibilidad. No minimizan la importancia del cuerpo o de la sexualidad en el desarrollo humano. Lo intrapsíquico no se opone a lo interpersonal: se complementan el uno con el otro.

El psicoanálisis relacional no es una escuela de pensamiento unificado, sino que constituye un grupo diverso de teorías que se enfocan en las relaciones personales, interpersonales e intrapersonales, y que destacan el énfasis en las dimensiones relacionales del desarrollo y del tratamiento. La teoría relacional es ecléctica y contemporánea; parte de la idea de que las relaciones, tanto internas como externas, reales e imaginadas, son su eje central.

\section{Cambio epistemológico}

Del positivismo y el objetivismo se pasa al constructivismo social. Se abandona la creencia de que el analista puede eliminar el impacto de su propia subjetividad, observar desde fuera del sistema. Con el constructivismo se enfatiza que el observar tiene un rol relevante en la configuración, construcción y organización de aquello que está siendo observado. Este cambio epistemológico promueve el escepticismo frente a lo objetivo y al conocimiento universal. Se pasa a considerar la subjetividad de los dos integrantes de la díada analítica. Se hace foco en la transferenciacontratransferencia como una coconstrucción mutua. 


\section{Elemento básico de la vida mental}

Uno de los puntos de anclaje del psicoanálisis relacional es la teoría del apego, la búsqueda del objeto como motivación básica del ser humano. La constitución de un sujeto (self) necesita de otro.

Fairbairn (apud Mitchell, 1993) argumenta que la libido no busca el placer, sino el objeto. La principal necesidad del niño es establecer una fuerte relación con otra persona. El motivo básico de la experiencia humana es la búsqueda y la conservación de un fuerte vínculo emocional con otra persona. Riera i Alibés (2011) dice que

los humanos nacemos con un cerebro que posee la capacidad potencial para la conexión intersubjetiva [...]. La consecuencia más negativa de la falta de maduración de las capacidades para la conexión intersubjetiva es que el pequeño no tendrá la capacidad de recurrir a los demás para regular sus propias emociones. (p. 223)

Desde la perspectiva relacional, el bebé no está gobernado por las pulsiones, sino que viene equipado para conectar con los objetos, construir con ellos sistemas relacionales progresivamente más complejos para conseguir una buena regulación mutua de las emociones.

Fairbairn (apud Rodríguez Sutil, 2007) plantea que el paciente debe haber sufrido deprivaciones importantes en su infancia, por lo cual es lógico que acuda a la terapia necesitado de aquellas relaciones personales de las que careció.

\section{Neurociencias}

El psicoanálisis relacional se nutre también de las investigaciones y los descubrimientos de las neurociencias respecto al desarrollo cerebral, la 
neuroplasticidad, la memoria procedural o implícita, el descubrimiento de las neuronas espejo, entre otros. Estos conocimientos tienen consecuencias en la concepción del desarrollo de la mente y en la postura clínica.

Las neuronas espejo desempeñan un importante papel en la capacidad humana para sentir lo que el otro está sintiendo. "Yo siento que tú sientes lo que yo siento» (Riera i Alibés, 2011, pp. 133, 179, 212). Son la base de la empatía, de la identificación y de la capacidad de mentalización.

La amnesia infantil hasta los 3 años de edad es entendida a la luz de la no disponibilidad del hipocampo, que no comienza su maduración hasta pasados los 18 meses de vida. La memoria implícita guarda las experiencias emocionales de los primeros meses, experiencias que no pueden ser recordadas explícitamente ni verbalizadas; solo se expresan como memoria emocional en los sentimientos y en el comportamiento. Al pensar la incidencia de estos elementos en el ámbito clínico, pasan a jerarquizase los componentes paraverbales, que crean la atmósfera emocional de la sesión y ayudan al paciente a ir modificando y ejercitando su memoria emocional.

\section{Cambios teóricos}

Se recupera la primera teoría freudiana sobre el trauma en cuanto a la importancia de lo ambiental en la subjetividad. Ferenczi (2016) fue el primero en señalar que el trauma no estaba solamente vinculado a lo sexual, y puso de relieve lo afectivo cuando el adulto no cumple su función protectora al ignorar las necesidades del niño. El trauma deja de organizarse exclusivamente alrededor de la sexualidad y adquieren una importancia equivalente la falla por exceso o déficit y la falta de sintonía, contención emocional y responsividad emocional adecuada entre el adulto y el niño.

Cambia la hermenéutica de la sospecha por la hermenéutica de la confianza en la escucha del paciente. Se cuestiona la universalidad de la 
fantasía de seducción: se escuchan, también, los eventos del ambiente en que se desarrolla el niño y se entienden las expresiones clínicas no solo como producto de la fantasía. Es otra forma de posicionarse con el paciente (investigación empática-introspectiva), la cual, sin dejar de lado la asimetría inherente a la relación profesional, ubica a ambos (paciente y terapeuta) como partícipes en los trastornos del desarrollo del tratamiento. Es así como deja de considerarse que no hay pacientes inanalizables, sino que ese paciente no funciona en análisis con ese analista. Se valora la personalidad del analista como uno de los factores que intervienen en el campo relacional entre analista y analizando.

Los sueños no son considerados únicamente como expresión de un impulso sexual reprimido: se les asigna una función de regulación y restauración de los procesos psíquicos. Se considera que los sueños también intentan integrar y organizar las experiencias afectivo-cognitivas actuales.

En el psicoanálisis relacional las emociones cobran relevancia, se exploran en el discurso del paciente y en todas las irrupciones del inconsciente. Las emociones guían y determinan el comportamiento de los seres humanos en todas sus dimensiones. Intervienen en los más diversos campos de acción humana: relaciones interpersonales, de pareja y familiares, de negocios, política, religión, economía, etcétera.

Son las emociones, de placer o de displacer, de esperanza o temor, de deseo o aversión, de amor o de odio, de amistad o de antipatía, de alegría o tristeza, y un larguísimo etcétera lo que constituye el meollo de nuestra vida psíquica. La capacidad de razonar, como la percepción, la memoria, etc., son los recursos de que disponemos para calibrar, filtrar, canalizar, inhibir o expresar nuestras emociones y, finalmente, comportarnos, mental y prácticamente, de una manera u otra manera. (Coderch de Sans y Plaza Espinosa, 2016, p. 23) 
Todo ser humano llega al mundo desvalido y, al mismo tiempo, provisto de un potencial de humanidad. Ese potencial se despliega en el contacto con otros seres humanos. Ese encuentro o desencuentro, tanto con las personas como con la sociedad que lo reciba, dará lugar a una gran variabilidad de resultados en ese ser que se hace humano.

\section{ENCUADRE EN PSICOANÁLISIS RELACIONAL}

El Diccionario introductorio de psicoanálisis relacional e intersubjetivo (León y Ortúzar, 2020) define el encuadre como un

Conjunto de reglas y constantes que permiten y facilitan el desarrollo del proceso terapéutico. Tradicionalmente, el psicoanálisis clásico ha abogado por un encuadre más fijo e inmóvil, mientras que el psicoanálisis relacional propone un encuadre elástico y flexible, resultante de la negociación mutua, dinámica y particular de cada díada terapeutapaciente. (Bass, 2007, apud León y Ortúzar, 2020, p. 202)

Se concibe el proceso psicoanalítico como algo que se genera en el encuentro de dos subjetividades: la del paciente y la del analista, que se influyen mutuamente. Es la creación compartida entre ellos de un ambiente seguro y ético, que a partir de un encuentro continuo permite, entre ambos, la exploración intensa y profunda de los aspectos inconscientes del paciente y la edición de situaciones novedosas, no vividas por el paciente (Nemirovsky, 2020).

El encuadre en esta perspectiva es flexible, antijerárquico, democrático y dialógico. Es una cocreación entre terapeuta y paciente que permite movimientos emocionales en un ambiente seguro, confiable y ético. 
Bregman Ehrenberg (2016) en su libro Al filo de la intimidad... nos proporciona numerosos ejemplos de este giro relacional.

\section{¿Cuál es la función del encuadre?}

Para el psicoanálisis freudiano, favorecer la regresión del paciente, necesaria para llevar a cabo el proceso psicoanalítico y la instauración de la neurosis de transferencia. Para el psicoanálisis relacional, que parte de la naturaleza social y relacional del ser humano, se entiende que el encuadre debe estimular y favorecer la progresión del funcionamiento psíquico del paciente.

Sabemos que los seres humanos poseemos múltiples self de acuerdo con los diversos contextos por los que ha transcurrido nuestra vida (Mitchell, 1993).

El reconocimiento del otro y el desarrollo del self son fenómenos interdependientes. La intersubjetividad, el reconocer al otro y sentirse reconocido por el otro, son el alimento del self. Por lo tanto, el espíritu del encuadre en el psicoanálisis relacional es el de estimular y favorecer la profundización de la intersubjetividad. (Coderch de Sans, 2018, p. 351)

Un encuadre para el psicoanálisis relacional no alude solamente a las coordenadas espaciotemporales y de actitud profesional en que se desarrolla el encuentro. Sabemos que entre paciente y analista se produce una ininterrumpida y mutua influencia (llamada clásicamente transferencia y contratransferencia), cocreada, por lo cual ningún analista es igual con todos sus pacientes, porque cada uno despierta en él diferentes estados de su self (conscientes y disociados). Por lo tanto, sería más adecuado plantear que entre ambos crean un encuadre interno, en cuyo marco transcurre esta peculiar interacción entre dos personas (Coderch de Sans, 2018). 
En el Río de la Plata, Baranger (2004) plantea:

La relación analítica es distinta conforme a la personalidad y las características de sus dos integrantes. Sabemos empíricamente que no todo paciente puede funcionar con cualquier analista, y recíprocamente, que no todo analista trabaja bien con cualquier paciente, por más que su análisis previo haya ampliado su capacidad de comprensión y su intuición. Es necesaria una adaptación mutua entre analista y paciente para permitir la formación del campo y de la fantasía inconsciente básica compartida. (p. 166)

Asimismo, en el Mediterráneo, Coderch de Sans (2015) plantea que, gracias a los conocimientos aportados por el sistema nerviosos central, en el encuadre psicoanalítico queda confirmada la innegable realidad de la influencia mutua de la díada analítica, sean cuales sean las intenciones y fantasías que posea cada uno de sus miembros:

Todo movimiento en el estado mental de cada uno de los componentes de la díada analítica, ya sea enunciado verbalmente en el contenido semántico de las palabras o en la prosodia del lenguaje, o sub-verbalmente a través de gestos y expresiones faciales o silencios, da lugar a que en el cerebro del otro se activen las mismas redes neuronales, de manera que el analista, con la atención vuelta hacia sí mismo, puede percibir no tan sólo las emociones y pensamientos que, de alguna manera, le provocan sus redes neuronales activadas y equivalentes a las que lo están en el paciente, sino también los procesos somatosensoriales correspondientes a tal activación de estructuras cerebrales, es decir, puede acompañar sensorialmente al paciente en las experiencias que está viviendo en la relación, gracias a lo cual podemos decir que este analista no únicamente 
comprende a su paciente, sino que, además, lo reconoce como la madre reconoce a su bebé. (Coderch de Sans, 2018, p. 334)

A la luz de estas observaciones, se podría plantear que el matiz diferente en cuanto a cómo se configura el campo analítico en la perspectiva relacional estaría dado por la importancia que cobran los procesos psíquicos implícitos en la díada analítica. Basta pensar en «lo sabido no pensado» de Bollas (1987). Se configura un diálogo implícito ente paciente y terapeuta por otros canales de expresión (no verbales), y allí nos encontramos con fenómenos como el enactment (De Souza y Anfusso, 2017), que son reveladores de lo que está sucediendo en la díada. Desde la perspectiva relacional, el enactment es una experiencia valiosa en el proceso terapéutico, en tanto se trata de instancias basadas en las emociones que cobran vida en la relación paciente-analista, en la cual intervienen ambos. Los enactment son una forma de comunicar las experiencias o estados emocionales más arcaicos, asimbólicos y no verbales, es decir que no pueden expresarse de otro modo. Es una comunicación, una escena donde pasa algo, se escenifica algo que no es consciente entre paciente y analista.

Siguiendo a Coderch de Sans (2018), el encuadre psicoanalítico (externo e interno) configura un campo donde es imposible tener todas las variables controladas. Se produce una reverberación de los estados mentales, pensamientos, sensaciones y emociones del paciente en la mente y en las sensaciones corporales del analista, y viceversa, del analista en la mente del paciente. Desde el psicoanálisis relacional, el analista debe aspirar a una actitud de entrega al otro sin dejar de ser él mismo, en un encuadre que estimule el diálogo abierto con todas las posibilidades, donde se despoje del temor a ser descubierto como persona, se ofrezca disponible para ser reconocido por el paciente y abandone la preocupación por mantener la fría distancia analítica, la neutralidad y el anonimato. 
Esto no significa que los analistas relacionales sean siempre simpáticos y laxos ni que hagan uso indiscriminado de la autorrevelación y el vale todo.

\section{ENCUADRE Y PANDEMIA}

Detengámonos ahora a reflexionar sobre la incertidumbre y los cambios que generó una situación excepcional en el 2020: la pandemia por el covid-19. La exhortación al confinamiento y el temor a lo desconocido, con potencial letal, hicieron que rápidamente adoptáramos el uso de tecnologías a nuestro alcance para poder continuar con nuestra tarea. El psicoanálisis vía videoconferencia modificaba el encuadre en el proceso analítico y no sabíamos cómo ello podría alterar el proceso y la identidad de nuestra práctica. Se produjo un ajuste activo a las necesidades del paciente, del analista y del contexto de pandemia.

El encuadre está en proceso de profundas transformaciones a la luz del psicoanálisis relacional y de la comunicación virtual y digital que el avance tecnológico ha proporcionado (León y Ortúzar, 2020). La pandemia nos impuso atender a nuestros pacientes a distancia. Debimos cambiar el encuadre, lo que derribó la ilusión de poseer algo seguro y estable al resguardo del consultorio en presencia corpórea.

Asistimos con anterioridad a los cambios en la cultura y en la subjetividad de los pacientes, que exigieron modificaciones en el encuadre, y confeccionamos a medida el encuentro con el que solicitaba nuestra ayuda. Con la pandemia, el encuentro se ha diseñado a través de diversas plataformas de videoconferencia: Skype, Zoom, teléfono, etc. El paciente elige y se arman diferentes encuadres, con imagen y sin imagen, mientras que otros no pudieron apropiarse de la copresencia virtual y no aceptaron esta nueva modalidad para continuar el proceso analítico. 
El encuentro se da en un mundo que incorpora la amenaza de muerte y la preocupación por la vida propia y de los otros. ¿Cómo dar amparo a las formas emergentes de ser y afrontar esta realidad, sin caer en una sobreadaptación que obture una transformación creativa de lo que estamos viviendo y haciendo?

Siguiendo a Gutiérrez (2020), podemos inferir el malestar subjetivo en estas relaciones de copresencia virtual. Este psicoanalista chileno nos propone pensar que lo que radicalmente viene a alterarse en la telecopresencia es la experiencia de otredad en el encuentro humano:

Cierto conjunto perceptual me indica que ahí hay otro, y me encuentro coordinándome, encontrándome con él. Por otro lado, la responsividad espontánea se encuentra entorpecida, el trasfondo psique somático que anticipa al otro, se encuentra con una respuesta ominosa, es familiar pero extranjera a la vez, y tenemos que resolver esta forma de comunicación silente, y lo hacemos mediante el uso de diversos mecanismos de defensa para lidiar con este malestar. La renegación o actitud de aceptar el objeto negando la falta es notorio. Al estar inmerso en la experiencia virtual avanzamos a alucinar negativamente el registro de la carencia. (Gutiérrez, 2020)

Perceptualmente la carencia desaparece: «Se reconstruye un otro a partir de una interacción por vías de la imagen y el sonido que reniega de la ausencia y al mismo tiempo se anticipa reflexivamente al otro, quedando, esperando aquello que nunca llega» (Gutiérrez, 2020).

Gutiérrez (2020) considera que la virtualidad tiene muchos beneficios prácticos, pero que también trae aparejada una serie de malestares subjetivos en el campo de las copresencialidades, que, a su entender, obedecen a esas condiciones estructurales que contravienen el modo de construir nuestra subjetividad a partir del encuentro humano. Nuestro self emerge 
de la experiencia de sostén físico y emocional de la madre en tanto ambiente. La experiencia temprana de maternaje opera como una estructura encuadrante para el sujeto. «Hacemos de lo externo el modo interno, y de cómo hemos sido tratados, un lienzo para la representación» (Gutiérrez, 2020). Estas consideraciones de la construcción de la subjetividad, a su entender, nos advierten de cuál es la población clínica con indicación o contraindicación de teleanálisis.

Asimismo, Coderch de Sans (2018) se interroga sobre dónde reside la posibilidad del cambio psíquico y la fuerza terapéutica del psicoanálisis desde la perspectiva relacional. En su respuesta (además de la personalidad del analista y el método o teorías que lo guían en su trabajo) considera la importancia de sintonizar psicobiológicamente con el paciente y lograr que se sienta reconocido en sus experiencias implícitas, no verbales y no simbólicas, de la misma manera que un bebé se siente reconocido por su madre.

Coderch de Sans (2018) afirma que es gracias a «la sintonización psicobiológica de las mentes corporeizadas de paciente y analista» (p. 56) que puede producirse el cambio y la modificación del inconsciente relacional. La sintonización psicobiológica es un concepto que se apoya en las investigaciones de Rizzolatti y Sinigaglia (2006) sobre las neuronas espejo.

Gallesse (2011) afirma que los humanos somos semejantes porque estamos equipados con el mismo substrato neuronal, que se activa cuando realizamos acciones o experimentamos emociones y sensaciones. A este mecanismo neuronal funcional lo llama simulación corporeizada. A través de él se logra una comprensión del otro, desde el interior, lo reconocemos dentro de nuestro propio organismo. Este mecanismo neuronal funcional nos permite compartir los estados emotivos, las necesidades y el sufrimiento. Poseemos un mecanismo espejo que nos permite reconocer inmediatamente al otro. Estas aportaciones de la neurofisiología y la 
neurobiología respecto a la captación de las emociones e intenciones del otro, gracias a la función del sistema nervioso central y a la simulación corporeizada, no derriban la utilidad de la comprensión basada en la reflexión. Por el contrario, abren una interrogante respecto a la posibilidad de su despliegue en el encuentro virtual con el paciente. El espejamiento en nuestro cerebro de las emociones y pensamientos de los pacientes: ¿es posible en la comunicación a través de los dispositivos electrónicos? ¿Corremos riesgo de pasar a una virtualización de la psicoterapia, con un self virtual y descorporeizado?

En el encuentro con el paciente se desarrolla otro diálogo subverbal a nivel implícito. Paciente y analista emiten señales sensoriales durante el diálogo: parpadeo, expresiones faciales y gestuales, movimientos corporales, expresión de los ojos en todas sus tonalidades, entonación y ritmo de la voz, entre otras, captadas en la comunicación en presencia física, necesaria para que se produzca la sintonización psicobiológica. Las percepciones somatosensoriales, origen de las experiencias implícitas, de las que derivan las explícitas, pueden estar muy restringidas en el intercambio con el paciente a través de los medios electrónicos. Está la imagen, pero no la experiencia vívida humana. Mediante la simulación corporeizada, junto al diálogo explícito paciente-terapeuta, se desarrolla otro diálogo subverbal a nivel implícito en ambos sentidos de la díada. El paciente también percibe internamente al analista. Es lo que Winnicott nos ha enseñado respecto a la díada madre-bebé y a cómo la madre percibe en su interior los estados mentales de su bebé y los refleja en su rostro, tono de voz, gestos...; asimismo el bebé sintoniza los estados emocionales de la madre (Anfusso e Indart, 2009).

¿El análisis online funciona con todos los pacientes? La experiencia clínica en pandemia nos fue mostrando la variabilidad de respuesta ante el nuevo dispositivo propuesto para continuar trabajando. Muchos pacientes se adaptaron fácilmente y lograron revelar a distancia relatos que 
de forma presencial no se habían producido. En otros casos, esto no fue posible, especialmente en aquellas situaciones en que el paciente requería una regresión profunda a la dependencia en su proceso de análisis.

Llegado el confinamiento, algunos analistas tenían experiencia de trabajar online, con encuadres reservados como medidas transitorias para mantener un tratamiento, de lo que no se hablaba mucho. Con la pandemia apareció la necesidad de continuar con nuestra tarea incluyendo un tercero, extraño para muchos de nosotros: el tercero tecnológico. Con él se instalaron varias interrogantes y preocupaciones, una de ellas: la confidencialidad, elemento fundante en la creación de un ambiente seguro y ético para desarrollar el encuentro con el paciente. Debimos asesorarnos y aprender los resguardos necesarios para continuar a través del encuentro online. Debimos tolerar las interferencias en la conectividad en el uso de diferentes plataformas y nos preguntamos si es lo mismo la ausencia del paciente por no conectarse. Movidos por la autoconservación, nos lanzamos a nadar en aguas extrañas, sin perder un modo de pensar psicoanalítico. ¿Fin del reinado del diván, del cara a cara, para dar cabida a otro encuadre en la práctica psicoanalítica: el psicoanálisis a distancia?

Estamos trabajando juntos cuando no estamos juntos, desarrollamos el psicoanálisis mediado vía internet, el ciberespacio ingresa en nuestra práctica y suscita discusiones respecto a su factibilidad e indicación. Existen diversas posiciones (positivas, neutras, negativas, moderadas) en torno a esta discusión sobre el análisis por videoconferencia (Gutiérrez, 2017), que consideran el impacto de las mediaciones virtuales en el proceso analítico. Gutiérrez (2020) nos propone pensar que los encuadres por videoconferencia producen

una bi-escisión entre diversos grados de reconocimiento/negativización de la ausencia del otro, por un lado, y la expectativa de la copresencia física por el otro, desmantelando con ello la experiencia unitaria 
prerreflexiva del ser aquí, ahora, conmigo, propia de las interacciones materialmente presentes.

A la luz de estas consideraciones, debemos preguntarnos qué paciente se beneficia con este nuevo encuadre a distancia y en qué casos no es pertinente. Nuevamente cobra relevancia lo singular en nuestra disciplina: depende del caso a caso.

El posicionarnos respecto a este asunto está mediatizado por la perspectiva teórica que nos acompaña en el encuentro con el paciente, los modos de entender la práctica clínica y los objetivos que consideremos tiene el análisis. Podemos estar preocupados por hallar la verdad, esclarecer o curar (objetivos que seguramente nos interesan en términos del proceso que vamos transitando). Vamos armando, construyendo entre ambos integrantes de la pareja terapéutica, intervenciones sucesivas que intentan acercarse a las vivencias de quien nos consulta y que tratan de abarcarla, dando rodeos metafóricos (Nemirovsky, 2019).

Desde la mirada que nos ofrece Winnicott con su teoría del desarrollo emocional, la no integración es un estado necesario para la emergencia del self (estados de unidad o ser being) (Sáinz Bermejo, 2017). Como lo plantea Mc Dougall (apud Gutiérrez, 2017), en un tratamiento psicoanalítico el acceso a los estados de no integración se relaciona «con la capacidad de un individuo de, sin miedo, poder flotar o entregarse a lo informe y la nada, y tener "tiempo para ser", con un énfasis en el ser en el lugar del hacer» (pp. 215-216).

Todos en esta pandemia estamos en un ser siendo, afrontando con creatividad el encuentro con los pacientes. A veces no logramos la sintonía anhelada en los nuevos encuadres y no podemos sobreponernos a la condición biescindida de la videoconferencia, lo que nos hace anhelar recuperar la conexión del encuentro presencial. 
El trabajo psicoanalítico a través de la videoconferencia tendrá que ser revisado no solo por las determinaciones estructurales del dispositivo, sino también por la capacidad de la díada paciente-analista de superar las peculiaridades del nuevo encuadre (escisión, interferencia, dificultad en la sincronización psicobiológica, dificultad en el acceso a estados de no integración, entre otras), sin olvidar las necesidades del paciente y sin apartarnos de los preceptos psicoanalíticos que nos guían. No se trata de rechazar o incluir ingenuamente estos dispositivos electrónicos en el encuentro con los pacientes, sino de explorar (acompañados por nuestras perspectivas teóricas y juicio clínico) cómo debemos llevar a cabo nuestra tarea con cada paciente. Nos movemos en la incertidumbre, buscando la manera distinta en cada caso de ser analista para cada paciente. El análisis a distancia nos reta a distinguir lo central de lo accesorio sin desnaturalizar nuestra práctica, sin perder nuestra identidad profesional.

En pandemia, la nube cibernética nos sostuvo y modificó el encuadre psicoanalítico, lo que nos permitió ser creativos y lanzarnos a explorar nuevas coconstrucciones. Comprobar su pertinencia y efectividad insumirá más tiempo y reflexión.

\section{$\S$}

\section{REFERENCIAS BIBLIOGRÁFICAS}

Anfusso, A. e Indart, V. (2009) ¿De qué hablamos cuando hablamos de Winnicott? Psicolibros

Ansermet, F. y Magistretti, P. (2006). A cada cual su cerebro: plasticidad neuronal e inconsciente. Katz. 
Aron, L. (2013). Un encuentro de mentes: mutualidad en psicoanálisis. Universidad Alberto Hurtado.

BARANGer, M. (2004). La teoría del campo. En L. Glocer Fiorini (comp.), El Otro de la trama intersubjetiva. Lugar.

Bowlby, J. (1988). Una base segura. Paidós.

Bregman Ehrenberg, D. (2016). Al filo de la intimidad. Extendiendo el alcance de la interacción psicoanalítica. Ágora Relacional.

Coderch de SANS, J. (2012). La práctica de la psicoterapia relacional. El modelo interactivo en el campo del psicoanálisis. Ágora Relacional.

Coderch de SAns, J. (2014). Avances en psicoanálisis relacional. Ágora Relacional.

Coderch de Sans, J. (2015). Las experiencias terapéuticas en el curso del proceso analítico desde la perspectiva de la no linealidad/complejidad. Temas de Psicoanálisis, 9. www.temasdepsicoanalisis.org/2015/01/31/ las-experiencias-terapeuticas-en-el-tratamiento-psicoanalitico-desdela-perspectiva-de-la-no-linealidadcomplejidad-2/

Coderch De SANS, J. (coord.). (2018). Las experiencias terapéuticas en el proceso psicoanalítico. Ágora Relacional.

Coderch de Sans, J. y Plaza Espinosa, A. (2016). Emoción y relaciones humanas. El psicoanálisis relacional como terapéutica social. Ágora Relacional.

De Souza, L. y Anfusso, A. (2017). Enactment: transferencia... ¿y después? Revista de Psicoterapia Psicoanalítica, 9(4), 5-18.

FERENCZI, S. (2016). Confusión de lengua entre los adultos y el niño. El lenguaje de la ternura y de la pasión. Revista de Psicoanálisis, 77, 39-51. GALLESSE, V. (2011). Neuronas espejo, simulación corporeizada y las bases neuronales de la identificación social. Clínica e investigación relacional, Revista electrónica de Psicoterapia, 5(1), 34-59. www.psicoterapiarelacional.es/Portals/0/eJournalCeIR/V5N1_2011/03_V_Gallese_ Neuronas-espejo-simulacion-corporeizada_CeIR_V5N1.pdf 
GutiéRREZ, L. (2017). ¿Silicio en el "Oro Puro”? Contribuciones teóricas y observacionales al teleanálisis por videoconferencia. The International Journal of Psychoanalysis, 3, 550-577. https://doi.org/ 10.1080/2057410X.2017.1649868

GutiéRREz, L. (2020). El individuo y el mundo en que vivimos [video]. www.youtube.com/watch?v=QLHuyhBfoFs\&t=3s

LeÓn, S. y ORTúZAR, B. (2020). Diccionario introductorio de psicoanálisis relacional e intersubjetivo. RIL.

MitcheLL, S. (1993). La matriz relacional. En Conceptos relacionales en psicoanálisis: Una integración (pp. 29-54). Siglo XXI.

Nemirovsky, C. (2019). Transformaciones en nuestra práctica. Clinica e investigación relacional, Revista electrónica de Psicoterapia, 13(1), 170-184. http:// dx.doi.org/10.21110/19882939.2019.130112

NemirovsKy, C. (2020). Psicoanálisis relacional [video]. https://m.youtube.com/ watch?v=BWdumeF_p3M\&feature=youtu.be

Riera i Alibés, R. (2011). La conexión emocional. Octaedro.

Rizzolatti, G. y Sinigaglia, C. (2006). Las neuronas espejo. Paidós.

Rodríguez SutiL, C. (2007). Epistemología del psicoanálisis relacional. Clínica e investigación relacional, Revista electrónica de Psicoterapia, 1, 9-41. www.psicoterapiarelacional.es/Portals/0/eJournalCeIR/V1N1/1_Rodriguez\%20 Sutil_Epistemologia\%20del\%20Psicoanalisis\%20Relacional_CeIR_ V1N1_2007.pdf

RodRíguez SutiL, C. (2010). Introducción a la obra de Ronald Fairbairn. Los origenes del psicoanálisis relacional. Ágora Relacional.

Sáinz Bermejo, F. (2017). Winnicott y la perspectiva relacional en el psicoanálisis. Herder. 
